\title{
IDEMPOTENT NOETHER LATTICES
}

\section{KENNETH P. BOGART}

In his paper, Abstract commutative ideal theory [2], Dilworth proved that a Noether lattice on which the multiplication is the meet operation is a finite Boolean algebra. This note proves that if the multiplication in a Noether lattice is idempotent $\left(A^{2}=A\right.$ for all $A$ in the lattice), then the lattice is a finite Boolean algebra. In a Noether lattice the term maximal element refers to a maximal nonidentity element (i.e. a coatom).

THEOREM. Let $L$ be a Noether lattice in which the maximal elements are idempotent. Then $L$ is a finite Boolean algebra.

Proof. Let $M$ be a maximal element of $L$. Then $M=M^{i}$ for all $i$. This gives

$$
\bigwedge_{i}\left(A \vee M^{i}\right)=A \vee M \text { for all } A \in L .
$$

Thus by Theorem 3.2 of [2], $A \bigvee M$ is the meet of all primary components of $A$ contained in $M$. We thus have that each element of $L$ is a meet of finitely many maximal elements of $L$. Since $L$ is modular and every element of $L$ is a meet of coatoms, $L$ is complemented. Then by Theorem 7.31 of [1], $L$ is a Boolean algebra. (The term "Noether lattice" has a different meaning in [1] from that in [2]. We are using the term as defined in [2].) Since $L$ has the ascending chain condition, $L$ is finite, and the theorem is proved.

Note that meet and multiplication coincide when multiplication is idempotent in a Noether lattice. To see this, observe that if

$$
\begin{gathered}
\left(M_{1} \wedge M_{2} \wedge \cdots \wedge M_{j}\right)\left(N_{1} \wedge N_{2} \wedge \cdots \wedge N_{k}\right) \\
<M_{1} \wedge \cdots \wedge M_{j} \wedge N_{1} \wedge \cdots \wedge N_{k},
\end{gathered}
$$

with $M_{i}$ and $N_{i}$ all maximal, then because each element of $L$ is a meet of maximal elements, there exists another maximal element $M$ such that

$$
\begin{aligned}
& \left(M_{1} \wedge M_{2} \wedge \cdots \wedge M_{j}\right)\left(N_{1} \wedge N_{2} \wedge \cdots \wedge N_{k}\right) \\
& \leqq M \wedge M_{1} \wedge \cdots \wedge M_{j} \wedge N_{1} \wedge \cdots \wedge N_{k}
\end{aligned}
$$

Then

Received by the editors October 10, 1968. 


$$
M_{1} M_{2} \cdots M_{j} N_{1} N_{2} \cdots N_{k} \leqq M .
$$

Thus since $M$ is prime, $M_{i}$ or $N_{i}<M$ for some $i$, and this is impossible.

REFERENCES

1. R. P. Dilworth and M. Ward, Residuated lattices, Trans. Amer. Math. Soc. 45 (1939), 335-354.

2. R. P. Dilworth, Abstract commutative ideal theory, Pacific J. Math. 12 (1962), 481-498.

Dartmouth College 